\title{
Le biais de temps immortel dans les études pharmacoépidémiologiques : définition, solutions et exemples
}

\author{
Jean-Luc Faillie ${ }^{1,2}$ et Samy Suissa $a^{3,4}$ \\ 1 Département de Pharmacologie Médicale et Toxicologie, CHRU de Montpellier, Montpellier, France \\ 2 Service de Pharmacologie Médicale et Clinique, Équipe de Recherche de Pharmacoépidémiologie, INSERM UMR 1027, \\ CHU de Toulouse, Faculté de Médecine Université Paul Sabatier, Toulouse, France \\ 3 Centre d'Épidémiologie Clinique, Institut de Recherches Lady Davis, Hôpital Général Juif, Montréal, Québec, Canada \\ 4 Département d'Épidémiologie, de Biostatistique et de Santé au Travail, Université McGill, Montréal, Québec, Canada
}

Texte reçu le 19 septembre 2014 ; accepté le 22 septembre 2014

\begin{abstract}
Mots clés :
biais de tempsimmortel ; pharmacoépidémiologie ; biais ; étude de cohorte ; bases de données

Résumé - Parmi les études observationnelles des effets des traitements médicamenteux des maladies chroniques, nombre d'entre elles ont conclu à des effets qui se sont avérés exagérés ou erronés. Parmi les biais responsables de ces erreurs, le biais de temps immortel, concernant la définition du facteur d'exposition étudié et des périodes d'exposition, occupe une place importante car il a généralement tendance à attribuer à tort un bénéfice important au médicament à l'étude (ou à exagérer un bénéfice réel). Dans cet article, nous définissons le mécanisme du biais de temps immortel, nous présentons les solutions possibles et nous illustrons ses conséquences au travers d'exemples d'études pharmacoépidémiologiques portant sur les effets des traitements médicamenteux.

\section{Keywords:}

immortal time bias; pharmacoepidemiology; biases; cohort studies; databases

Abstract - Immortal Time Bias in Pharmacoepidemiological Studies: Definition, Solutions and Examples. Among the observational studies of drug effects in chronic diseases, many of them have found effects that were exaggerated or wrong. Among bias responsible for these errors, the immortal time bias, concerning the definition of exposure and exposure periods, is relevantly important as it usually tends to wrongly attribute a significant benefit to the study drug (or exaggerate a real benefit). In this article, we define the mechanism of immortal time bias, we present possible solutions and illustrate its consequences through examples of pharmacoepidemiological studies of drug effects.
\end{abstract}

Abréviations : voir en fin d'article.

\section{Introduction}

Les essais thérapeutiques randomisés à la disposition de la communauté médicale présentent souvent des limites pour étudier les effets des médicaments à long terme, sur des critères cliniques forts et en comparaison aux traitements de référence appropriés. Cela est particulièrement le cas dans le champ des maladies chroniques et ainsi, une place importante est laissée aux études observationnelles des effets médicamenteux pour obtenir des informations utiles aux choix thérapeutiques. Cependant, de nombreuses études pharmacoépidémiologiques réalisées dans ce contexte ont conclu à des effets qui se sont avérés exagérés ou erronés lorsque des études randomisées adéquates ont été réalisées. Les biais responsables de ces erreurs peuvent être difficilement contrôlables (c'est le cas des effets de confusion lorsqu'on ne dispose pas des données pertinentes) mais des biais peuvent aussi être introduits par de mauvais choix méthodologiques. Le biais de temps immortel fait partie de cette dernière catégorie car il concerne essentiellement la définition du facteur d'exposition étudié. Ce biais, décrit initialement en 2003 dans le contexte du traitement de la bronchopneumopathie chronique obstructive (BPCO) par les corticostéroïdes inhalés (CSI), s'est propagé de façon remarquable ces dernières années. ${ }^{[1,2]}$ Dans ce manuscrit, nous définissons le mécanisme du biais de temps immortel, nous présentons les 


\begin{tabular}{|c|c|c|c|c|c|}
\hline $\begin{array}{l}\text { Patients } \\
\text { non traités }\end{array}$ & $\begin{array}{l}\text { Début du } \\
\text { suivi }\end{array}$ & & \multicolumn{2}{|c|}{$\begin{array}{l}\text { Évènement } \\
\text { d'intérêt }\end{array}$} & $\begin{array}{l}\text { Non exposés (NE) } \\
\square \text { Exposés (E) }\end{array}$ \\
\hline \multirow[t]{3}{*}{ Patients traités } & $\begin{array}{l}\text { Début du } \\
\text { suivi }\end{array}$ & \multicolumn{2}{|c|}{$\begin{array}{l}\text { Début du } \\
\text { traitement }\end{array}$} & \multicolumn{2}{|l|}{$\begin{array}{l}\text { Évènement } \\
\text { d'intérêt }\end{array}$} \\
\hline & & & \multirow[b]{2}{*}{ Risque relatif (RR) non biaisé } \\
\hline & Porr & classifiee & & & \\
\hline \multirow[t]{2}{*}{$\begin{array}{l}\text { Patients traités } \\
\text { Cas } n^{\circ} 1: \text { biais } \\
\text { de classification }\end{array}$} & $\begin{array}{l}\text { Début du } \\
\text { suivi }\end{array}$ & \multicolumn{2}{|c|}{$\begin{array}{l}\text { Début du } \\
\text { traitement }\end{array}$} & $\begin{array}{c}\text { Évènement } \\
\text { d'intérêt } \\
\downarrow\end{array}$ & $\begin{array}{l}>\text { Personnes-Temps E : \Incidence E } \\
\searrow \text { Personnes-Temps NE : >Incidence NE }\end{array}$ \\
\hline & \multicolumn{2}{|c|}{$\begin{array}{c}\text { Période immortelle } \\
\text { mal classifiée }\end{array}$} & & & Donc, sous-estimation du Risque relatif (RR) \\
\hline \multirow{2}{*}{$\begin{array}{l}\text { Patients traités } \\
\text { Cas } n^{\circ} 2 \text { : biais } \\
\text { de sélection }\end{array}$} & \multirow{2}{*}{\multicolumn{2}{|c|}{$\begin{array}{l}\text { Période immortelle } \\
\text { exclue }\end{array}$}} & & $\begin{array}{l}\text { Évènement } \\
\text { d'intérêt }\end{array}$ & $\searrow$ Personnes-Temps NE : $>$ Incidence NE \\
\hline & & & & & Donc, sous-estimation du Risque relatif (RR) \\
\hline
\end{tabular}

Fig. 1. Le biais de temps immortel survient lorsque la période entre le début du suivi et le début de l'exposition est considérée comme exposée (cas $n^{\circ} 1$ ) ou est exclue (cas $\left.\mathrm{n}^{\circ} 2\right)$. D'après ${ }^{[26]}$

solutions possibles et nous illustrons ses conséquences au travers d'exemples d'études pharmacoépidémiologiques portant sur les effets des traitements médicamenteux.

\section{Définitions}

En épidémiologie, le temps immortel se réfère à une période de suivi au cours de laquelle l'évènement d'intérêt ne peut survenir. ${ }^{[3,4]}$ Nous allons aborder cette notion par un exemple éloigné de la pharmacoépidémiologie mais qui illustre bien ce concept : une étude (publiée en 2001 dans la revue Annals of Internal Medicine) a suggéré que l'espérance de vie des acteurs qui reçoivent un Oscar serait de quatre ans supérieure à celle des autres acteurs. ${ }^{[5]}$ L'exposition est ici définie par le fait de recevoir un Oscar au cours de sa carrière d'acteur, et l'espérance de vie est mesurée par l'âge au décès. Une période immortelle est introduite car les acteurs oscarisés ont dû obligatoirement survivre jusqu'à leur Oscar et n'ont pu décéder avant. Autrement dit, dans le groupe des acteurs oscarisés, par définition, aucun décès n'a pu survenir entre leur naissance et leur Oscar. Cette période immortelle compte pourtant dans la mesure de l'espérance de vie des acteurs oscarisés et leur donne un avantage vis-à-vis des acteurs non oscarisés. La différence de longévité observée était ainsi artificielle. On peut retrouver cette erreur à chaque fois que l'on compare des populations selon des facteurs nécessitant un temps d'expérience différentiel : les évêques paraissent vivre plus longtemps que les curés, les juges que les avocats ou les généraux que les lieutenants. ${ }^{[6]}$

Dans les études de cohorte sur les effets de médicaments (notamment les médicaments utilisés au long cours dans les maladies chroniques), on peut observer une période de temps immortel lorsque, dans le groupe exposé au traitement à l'étude, un délai sépare le début du suivi et le moment où le patient est exposé. Les sujets exposés devant survivre (sans évènement) à cette période pour être considérés comme tels, on ne retrouve aucun évènement pour ces sujets pendant cette période : ce sont des personnes-temps « immortelles ». ${ }^{[2]}$ Pendant ce temps de suivi sans évènement, ces sujets ne sont pas (encore) exposés et devraient donc participer au groupe non exposé (figure 1). Le biais de temps d'immortalité survient soit lorsque cette période est attribuée à tort au groupe exposé (biais de classement, figure 1 , cas $\mathrm{n}^{\circ} 1$ ), soit lorsqu'elle est exclue de l'analyse (biais de sélection, figure 1 , cas $n^{\circ} 2$ ). Dans les deux cas, le biais procure un avantage au groupe exposé se traduisant par une sous-estimation du risque relatif c'est-à-dire une tendance à montrer un effet protecteur du traitement étudié. Dans le cas $n^{\circ} 1$, le mauvais classement des personnes-temps immortelles sousestime l'incidence du groupe exposé en rajoutant au dénominateur les personnes-temps immortelles et surestime l'incidence du groupe non exposé, au dénominateur de laquelle manquent ces personnes-temps immortelles. Dans le cas $n^{\circ} 2$, seule l'incidence dans 
le groupe non exposé est surestimée par l'exclusion des personnestemps immortelles. L'analyse correcte devrait employer des méthodes adéquates pour attribuer ces personnes-temps immortelles au groupe non exposé. ${ }^{[2]}$ Ce biais peut survenir dans toute situation où le suivi du patient et le traitement ne débute pas au même moment. On retrouve fréquemment la situation où la définition du statut exposé implique que le sujet reçoive le traitement pendant une durée minimale au cours du suivi (par exemple, deux prescriptions consécutives en deux mois), si cette période n'est pas considérée non-exposée, les résultats peuvent être biaisés en faveur du traitement.

\section{Solutions}

Parmi les solutions pour éviter le biais de temps immortel, on peut, en premier lieu, éviter l'ajout d'une période de temps immortel en faisant débuter le suivi des patients exposés et non exposés à partir de la fin de la période immortelle identifiée. ${ }^{[3]}$ Cette méthode est simple mais introduit une sélection des patients pouvant compromettre la représentativité de la cohorte. ${ }^{[7]}$ En effet, seuls les survivants de la période immortelle seront analysés. De plus, cette méthode nécessite que l'on puisse déterminer la fin de la période immortelle chez les non exposés. La solution à privilégier reste de garder la période de temps immortel en l'attribuant correctement à groupe non exposé. Pour cela, il faut éviter d'utiliser un modèle statistique où l'exposition est considérée comme fixe depuis le début du suivi et utiliser plutôt un modèle considérant l'exposition comme variable dépendante du temps. Ainsi, cette approche permet de considérer les patients comme non exposés depuis le début du suivi jusqu'à la date où ils remplissent les critères définissant l'exposition et de les considérer exposés après cette date. ${ }^{[2]}$ Une autre solution possible est d'utiliser l'approche des études castémoins nichées avec appariement sur la durée du suivi. ${ }^{[8]}$ L'exposition y est mesurée chez les cas et les témoins à la date index (date de l'évènement pour les cas et date correspondante à la même durée de suivi chez les témoins appariés). Ainsi, un cas survenant entre le début du suivi et le début du traitement sera correctement considéré comme non exposé. Il faut toutefois s'assurer que la cohorte soit bien définie notamment que la définition d'entrée dans la cohorte soit identique pour tous les sujets.

\section{Exemples}

\subsection{Traitement hormonal substitutif et risque cardiovasculaire}

Plusieurs études observationnelles avaient suggéré une réduction importante du risque cardiovasculaire des femmes ménopausées sous traitement hormonal substitutif (THS). Dans la première de ces études (en 1997), le THS était associé à une réduction spectaculaire de $62 \%$ de la mortalité après une coronarographie. ${ }^{[9]}$ Dans cette cohorte, les femmes qui recevaient un THS à n'importe quel moment après le début du suivi (coronarographie) étaient considérées exposées pour la totalité du suivi, engendrant ainsi des années de temps immortel mal classifié (figure 1 , cas $\mathrm{n}^{\circ} 1$ ). Plus tard, l'essai randomisé Women's Health Initiative montra l'absence de protection cardiovasculaire du THS, suggérant même un sur-risque. ${ }^{[10]}$

\subsection{Metformine et incidence des cancers}

Le potentiel rôle protecteur de la metformine sur l'incidence des cancers a fait l'objet de nombreuses publications. Des métaanalyses d'études observationnelles ont rapporté une réduction significative de plus de $30 \%$ du risque de cancer associé à l'utilisation de la metformine chez des sujets diabétiques. ${ }^{[11,12]}$ Parmi ces études, nombreuses étaient celles qui souffraient, à des degrés divers, d'un biais de temps immortel. ${ }^{[13]}$ Par exemple, une étude de cohorte réalisée sur une base des données de santé taiwanaise montrait une réduction de $88 \%$ de l'incidence des cancers chez les patients qui avaient reçu au moins deux prescriptions de metformine entre 2000 et $2007 .{ }^{[14]}$ Pour être exposés, les sujets ne pouvaient donc présenter un cancer avant la deuxième prescription : la période précédant cette deuxième prescription était immortelle mais comptait à tort pour le groupe exposé. Le biais participait ainsi au spectaculaire effet observé. Dans une autre étude de cohorte, utilisant le registre du diabète de Hong Kong entre 1996 et 2005, l'utilisation de la metformine était associée à des réductions significatives de $49 \%$ à $70 \%$ (selon les niveaux de valeurs du HDLcholestérol). ${ }^{[15]}$ Ici, les utilisateurs de metformine étaient suivis à partir du début de leur traitement par metformine et comparés aux non utilisateurs suivis à partir du diagnostic de leur diabète. Ainsi, pour les patients traités, la période entre le diagnostic et le traitement par metformine était donc exclue de l'analyse alors que ces personnes-temps auraient dû être attribuées au groupe non traités (figure 1, cas $n^{\circ}$ 2). ${ }^{[13]}$ Le rôle protecteur de la metformine dans la survenue de cancer semble avoir été pour le moins exagéré dans ces études observationnelles. Les résultats des études de cohorte qui ont tenté d'éviter les biais temporels ne montraient pas cet effet protecteur $^{[13]}$ et plusieurs méta-analyses d'essais randomisés qui ont étudié cette question ne retrouvent pas non plus de lien entre l'utilisation de metformine et la survenue ou la mortalité par cancer. ${ }^{[16-18]}$ Toutefois, motivés par les résultats prometteurs des études de cohorte, de grands essais de phase III ont été débutés pour évaluer la metformine en prévention secondaire du cancer de la prostate localisé $^{[19]}$ et du cancer du sein. ${ }^{[20]}$

\subsection{Corticostéroïdes inhalés et BPCO}

L'utilisation des corticostéroïdes inhalés (CSI) dans la prise en charge de la BPCO a largement été débattue. A partir de bases de 
données médico-administratives, plusieurs grandes études observationnelles ont étudié l'effet des CSI sur la mortalité de patients atteints de BPCO et ont conclu à une réduction très importante de la mortalité et de la morbidité (jusqu'à plus de $50 \%$ ). ${ }^{[21,22]}$ Une fois encore, un temps immortel n'avait pas été correctement pris en compte dans ces études. Une des premières études a été réalisée sur une base de données ontarienne et montrait une réduction significative de $29 \%$ de la mortalité associée à l'utilisation de CSI par rapport à la non-utilisation chez des patients atteints de BPCO. ${ }^{\text {[23] }}$ Dans cette étude, les patients étaient considérés comme exposés s'ils recevaient un CSI à tout moment au cours d'une période de 90 jours suivant leur hospitalisation. Ainsi, un temps immortel était introduit durant la période habituellement à risque entre la sortie de l'hôpital et la première prescription de corticoïdes inhalés et cette période comptait pout le groupe exposé aux CSI. Sur les 9000 personnes-années du groupe exposé, ce temps immortel comptabilisait 1500 personnes-années et lorsque l'on les attribuait au groupe non exposé, le risque relatif devenait égal à $1,0 .{ }^{[24]}$ Les auteurs de ces études observationnelles avaient appelé à la mise en place d'études randomisées pour prouver l'efficacité des CSI. C'est ainsi que le grand essai TORCH a été réalisé : comparé au placebo, l'utilisation de CSI ne montrait pas de bénéfices en termes de mortalité des patients atteints de BPCO (hazard ratio 1,06; intervalle de confiance à $95 \%: 0,89-1,27) .{ }^{[25]}$

\subsection{Statines et progression du diabète de type 2}

Une étude de cohorte utilisant les données de la base médicoadministrative du Saskatchewan au Canada a inclus 10996 nouveaux utilisateurs de médicaments antidiabétiques oraux entre 1991 et 1996 et a rapporté une diminution significative de $26 \%$ du risque de nécessiter un traitement par insuline chez les patients qui utilisaient une statine. ${ }^{[26]}$ Ce résultat était plutôt contre-intuitif étant donné que la nécessité d'une statine pourrait plutôt être associée à une maladie plus avancée et un diabète moins bien contrôlé. En fait, dans cette étude, les utilisateurs d'une statine pendant au moins un an au cours du suivi étaient considérés exposés depuis le début du suivi, ceci générant évidement une période de temps immortel mal classifiée. D'autres auteurs ont réalisé une analyse correcte de ces données en utilisant un modèle prenant en compte l'exposition dépendante du temps et ont montré au contraire une augmentation du risque de progression du diabète (hazard ratio 1,97 ; intervalle de confiance à $95 \%: 1,53$ à 2,52). Ces auteurs ont même montré que la méthode utilisée dans l'étude initiale aurait pu permettre d'obtenir des effets protecteurs des anti-inflammatoires non stéroidiens et des inhibiteurs de la pompe à protons sur la progression du diabète. Ces effets disparaissaient lorsque la méthode correcte était employée. ${ }^{[27]}$

\subsection{Statines et fractures osseuses}

Un autre effet bénéfique des statines avait été suggéré par une étude cas-témoin nichée au sein d'une cohorte de 91611 patients dans la base de données britannique du General Practice Research Database (GPRD) : une réduction de $45 \%$ du risque de fracture osseuse était rapportée chez les utilisateurs de statines. ${ }^{[28]}$ Nous avons vu que l'utilisation d'une étude cas-témoins nichée permet d'éviter le biais de temps immortel si les cas et les témoins sont appariés sur la durée du suivi. Ceci nécessite que la définition d'entrée dans la cohorte soit identique pour tous les sujets. Ce n'était pas le cas dans cette étude : les utilisateurs de statines débutaient leur suivi au moment de leur prescription de statine alors que les sujets non exposés débutaient le suivi un an après être enregistrés dans la base. Pour les sujets exposés, aucune fracture ne pouvait donc survenir entre l'année après l'enregistrement et le traitement par statine : cette période était donc immortelle et exclue de l'analyse alors qu'elle aurait dû compter pour le groupe non exposé. ${ }^{[2]}$ En reprenant la même base de données, d'autres analyses qui évitaient ce biais ne retrouvaient aucun lien entre l'utilisation de statine et les fractures osseuses. ${ }^{[29,30]}$

\section{Conclusions}

De nombreux autres exemples d'études pharmacoépidémiologiques pourraient être présentés pour illustrer ce biais de temps immortel. ${ }^{[2]}$ Un tel biais peut avoir des conséquences fâcheuses en attribuant des bénéfices spectaculaires à un médicament qui en est en réalité dépourvu (ou bien qui n'en présente pas autant que ce qui est avancé) et ainsi tromper patients et soignants dans leur choix thérapeutiques. Nous avons vu par ailleurs que de telles études pouvaient permettre l'autorisation d'essais cliniques inutiles et coûteux. Tout lecteur critique d'étude d'observationnelles sur les effets des médicaments doit rester vigilant afin de détecter la présence de biais de temps immortel, a fortiori lorsque les résultats sont en faveur d'un large bénéfice apporté par le médicament.

\section{Conflits d'intérêts. Aucun.}

Financement. Aucune aide financière n'a été nécessaire à la réalisation de ce travail.

Abréviations. BPCO : bronchopneumopathie chronique obstructive; CSI : corticostéroïdes inhalés; GPRD : General Practice Research Database; HDL : high density lipoprotein ; RR : risque relatif ; THS : traitement hormonal substitutif. 


\section{Références}

1. Suissa S. Effectiveness of inhaled corticosteroids in chronic obstructive pulmonary disease: immortal time bias in observational studies. Am J Respir Crit Care Med 2003; 168(1): 49-53

2. Suissa S. Immortal time bias in observational studies of drug effects. Pharmacoepidemiol Drug Saf 2007; 16(3): 241-9

3. Rothman KJ, Greenland S. Cohort studies. In: Rothman KJ, Greenland 8 S, eds. Modern epidemiology. 2nd ed. Lippincott-Raven 1998: 79-91

4. Walker AM. Observation and inference: an introduction to the methods of epidemiology. Epidemiology Resources, Inc.: Newton Lower Falls, MA, 1991

5. Redelmeier DA, Singh SM. Survival in Academy Award-winning actors and actresses. Ann Intern Med 2001; 134(10): 955-62

6. Sylvestre MP, Huszti E, Hanley JA. Do OSCAR winners live longer than less successful peers? A reanalysis of the evidence. Ann Intern Med 2006 Sep 5; 145(5): 361-3; discussion 92

7. Zhou Z, Rahme E, Abrahamowicz M, et al. Survival bias associated with time-to-treatment initiation in drug effectiveness evaluation: a comparison of methods. Am J Epidemiol 2005; 162(10): 1016-23

8. Suissa S. Novel approaches to pharmacoepidemiology study design and statistical analysis. In: Strom BL, ed. Pharmacoepidemiology. 4th ed. John Wiley, 2005: 811-29

9. Sullivan JM, El-Zeky F, Vander Zwaag R, et al. Effect on survival of estrogen replacement therapy after coronary artery bypass grafting. Am J Cardiol 1997; 79(7): 847-50

10. Rossouw JE, Anderson GL, Prentice RL, et al. Risks and benefits of estrogen plus progestin in healthy postmenopausal women: principal results from the Women's Health Initiative randomized controlled trial. JAMA 2002; 288(3): 321-33

11. Decensi A, Puntoni M, Goodwin P, et al. Metformin and cancer risk in diabetic patients: a systematic review and meta-analysis. Cancer Prev Res (Phila) 2010; 3(11): 1451-61

12. Noto $\mathrm{H}$, Goto $\mathrm{A}$, Tsujimoto $\mathrm{T}$, et al. Cancer risk in diabetic patients treated with metformin: a systematic review and meta-analysis. PLoS One 2012; 7(3): e33411

13. Suissa S, Azoulay L. Metformin and the risk of cancer: time-related biases in observational studies. Diabetes Care 2012; 35(12): 2665-73

14. Lee MS, Hsu CC, Wahlqvist ML, et al. Type 2 diabetes increases and metformin reduces total, colorectal, liver and pancreatic cancer incidences in Taiwanese: a representative population prospective cohort study of 800,000 individuals. BMC Cancer 2011; 11: 20

15. Yang X, So WY, Ma RC, et al. Low HDL cholesterol, metformin use, and cancer risk in type 2 diabetes: the Hong Kong Diabetes Registry. Diabetes Care 2011; 34(2): 375-80
16. Thakkar B, Aronis KN, Vamvini MT, et al. Metformin and sulfonylureas in relation to cancer risk in type II diabetes patients: a meta-analysis using primary data of published studies. Metabolism 2013; 62(7): 922-34

17. Franciosi M, Lucisano G, Lapice E, et al. Metformin therapy and risk of cancer in patients with type 2 diabetes: systematic review. PLoS One 2013; 8(8): e71583

18. Stevens RJ, Ali R, Bankhead CR, et al. Cancer outcomes and all-cause mortality in adults allocated to metformin: systematic review and collaborative meta-analysis of randomised clinical trials. Diabetologia 2012; 55(10): 2593-603

19. The Metformin Active Surveillance Trial (MAST) Study. http://clinicaltrials.gov/ct2/show/NCT01864096 Consulté le 22 septembre 2014

20. A phase III randomized trial of metformin vs placebo in early stage breast cancer. http://clinicaltrials.gov/show/NCT01101438 Consulté le 22 septembre 2014

21. Suissa S. Inhaled steroids and mortality in COPD: bias from unaccounted immortal time. Eur Respir J 2004; 23(3): 391-5

22. Soriano JB, Vestbo J, Pride NB, et al. Survival in COPD patients after regular use of fluticasone propionate and salmeterol in general practice. Eur Respir J 2002; 20(4): 819-25

23. Sin DD, Tu JV. Inhaled corticosteroids and the risk of mortality and readmission in elderly patients with chronic obstructive pulmonary disease. Am J Respir Crit Care Med 2001; 164(4): 580-4

24. Suissa S. Randomized trials built on sand: examples from COPD, hormone therapy, and cancer. Rambam Maimonides Med J 2012; 3(3): e0014

25. Calverley PM, Anderson JA, Celli B, et al. Salmeterol and fluticasone propionate and survival in chronic obstructive pulmonary disease. $\mathrm{N}$ Engl $\mathrm{J}$ Med 2007; 356(8): 775-89

26. Yee A, Majumdar SR, Simpson SH, et al. Statin use in Type 2 diabetes mellitus is associated with a delay in starting insulin. Diabet Med 2004; 21(9): 962-7

27. Levesque LE, Hanley JA, Kezouh A, et al. Problem of immortal time bias in cohort studies: example using statins for preventing progression of diabetes. BMJ 2010; 340: b5087

28. Meier CR, Schlienger RG, Kraenzlin ME, et al. HMG-CoA reductase inhibitors and the risk of fractures. JAMA 2000; 283(24): 3205-10

29. van Staa TP, Wegman S, de Vries F, et al. Use of statins and risk of fractures. JAMA 2001; 285(14): 1850-5

30. Hennessy S, Strom BL. Statins and fracture risk. JAMA 2001; 285(14): $1888-9$

Correspondance et offprints: Jean-Luc Faillie, Centre Régional de Pharmacovigilance du Languedoc Roussillon, Département de Pharmacologie Médicale et Toxicologie, CHRU Montpellier, 371 avenue du doyen Gaston Giraud, 34295 Montpellier, France.

E-mail : jl-faillie@chu-montpellier.fr 